\title{
Lifestyle Risk Factor Assessment Through WHO STEP Approach in Tabriz, Iran
}

\author{
Mina Golestani' \\ Homayoun Sadeghi- \\ Bazargani $^{2}$ \\ Mohammad Saadati ${ }^{1}$ \\ Mostafa Farahbakhsh (D) $^{3}$ \\ Koustuv Dalal $\mathbb{1 D}^{4,5}$ \\ 'Road Traffic Injury Research Center, \\ Tabriz University of Medical Sciences, \\ Tabriz, Iran; ${ }^{2}$ Road Traffic Injury Research \\ Center, Statistics and Epidemiology \\ Department, Tabriz University of Medical \\ Sciences, Tabriz, Iran; ${ }^{3}$ Clinical Psychiatry \\ Research Center, Tabriz University of \\ Medical Sciences, Tabriz, Iran; \\ ${ }^{4}$ Department of Public Health Science, \\ School of Health Sciences, Mid Sweden \\ University, Sundsvall, Sweden; ${ }^{5}$ School of \\ Medicine and Health Care, Al-Farabi \\ Kazakh National University, Almaty, \\ Kazakhstan
}

Correspondence: Koustuv Dalal Department of Public Health Science, School of Health Sciences, Mid Sweden University, Sundsvall, Sweden

Tel +46707I40I22

Email koustuv2010@hotmail.com
Background: The aim of this study was to assess the lifestyle behaviour and risk factors for lifestyle-related diseases in East Azerbaijan province, Iran.

Methods: A household study using a two-stage cluster sampling method was performed. Tabriz city was randomly selected for data collection among five geographic regions in the East-Azerbaijan province. Short WHO-STEP and Ultra-short version of Socio-Economic Status assessment questionnaire were used. Six hundred households were asked to respond to the STEP questionnaire.

Results: A total of 1196 people have participated in the study. People with higher socioeconomic status consumed more fruits, vegetables and fish than the people with lower socioeconomic status. People with academic education less likely to be hypertensive compared to people with non-academic education. People with a medium socioeconomic status are less likely to be hypertensive than people with high socioeconomic status. The majority of participants had poor dietary habits. In this study, $17.22 \%, 7.53 \%$ and $4.35 \%$ of respondents had hypertension, diabetes and depression, respectively.

Conclusion: Considering that lifestyle-related risk factors are common among people. Due to the direct link between lifestyle and the occurrence of many chronic diseases, campaigns for and training programs to implement healthy lifestyle habits are recommended.

Keywords: lifestyle, risk factor, STEP approach, assessment

\section{Introduction}

Lifestyle is the way of living of individuals, families (households), and societies, which they manifest in coping with their physical, psychological, social, and economic environments on a day-to-day basis. ${ }^{1}$ Studies conducted by the World Health Organization (WHO) showed that about $60 \%$ of Quality of Life is associated with lifestyle and health behaviours. Also, 53\% of mortalities are related to these issues. ${ }^{2}$ Studies have identified specific types of behaviours that contribute to the development of Non-Communicable Diseases (NCDs). Lifestyle diseases occurred primarily due to inappropriate relationships between a person's daily routine and environment. The main factors contributing to lifestyle diseases include bad nutritional habits, physical inactivity, wrong body posture, and disturbed biological clock. $^{3,4}$ NCDs have been already gained significant importance in developed countries and are rapidly turning into a significant public health challenge in developing countries. Not all, but a large proportion of lifestyle disease mortality, particularly those from Cardiovascular Diseases (CVDs) and Lung cancer, can be are prevented by making changes in modifiable risk factors. ${ }^{5}$ A healthy lifestyle reduces morbidity and premature death. When a healthy lifestyle is adopted, a more 
positive role model is provided to other people in the family, particularly for children. ${ }^{6}$ Out of the three major groups of diseases and injuries (pre-transition, NCDs, and accidents and injuries), NCDs have caused the highest disease burden in Iran, $45 \%$ for men and $33 \%$ for women. According to the results obtained in the fifth round of survey of NCD risk factor surveillance program in Iran (2010), in both genders, only $4 \%$ in the age group of 15-44 years and 1\% in the age group of 45-64 years had no CVDs risk factors. ${ }^{4}$ Achieving the results of such studies on the risk factors of NCDs by using the WHO STEP approach can examine the relationship between socioeconomic factors and lifestyle, which can ultimately be helpful for health researchers and policymakers to assess health status in regions of the country and to plan to health promotion. The purpose of the current study was to assess the lifestyle behaviour and the risk factors of lifestyle-related disease in East-Azerbaijan province, Iran.

\section{Methods}

A household study using a two-stage cluster sampling method with Probability Proportional to Size (PPS) approach was conducted in East-Azerbaijan province, Iran. East Azerbaijan province has a total population of around 3900,000 people (based on the 2016 census).

One county was selected randomly as the representative of five geographical parts (central, northwest, southwest, northeast, and southeast). Six selected geographic regions were Tabriz city as the province capital, the city of Osko in central, Marand in the northwest, Varzeghan in the northeast, Bonab in the southwest, and Mianeh southeast part of East Azerbaijan province. One hundred twenty clusters were allocated based on proportion to population size (PPS), 2016 census. The trained interviewers interviewed the head of households or the housewife. In case of absence of persons in the households, we approached up to three times for data collection. A household with a residential time of fewer than six months in each area was excluded.

Short WHO-STEP and Ultra-short version of Socioeconomic Status assessment questionnaire (SESIran) questionnaires were used for data collection. STEP questionnaire included two main parts: demographic data such as age, gender, education, insurance, household dimension and the second part comprising of 12-Items on lifestyle such as smoking, vegetable and fruits consumption, daily diet style and physical activity. The questionnaire validity (Kappa coefficient $=0.94$ ) and reliability
(Cronbach-Alpha=0.98, ICC=0.94; CI: 0.87-0.97) were confirmed. Descriptive statistics, including frequency, mean \pm standard deviation (SD) and inferential statistics based on data normality, were utilised.

This study was conducted in accordance with the Declaration of Helsinki. The ethical committee approved the study of Tabriz University of Medical Sciences (TUOMS) (ethical code: TBZMED.REC.1394.35). Written informed consent was obtained from all participants.

\section{Results}

Total 1196 people have participated in the study with a mean age of $42.12 \pm 15.43$ years. Among them, 386 (32.27\%) were men and $810(67.73 \%)$ were women (Table 1$)$. Mean age of women $(40.95 \pm 14.77)$ was significantly $(p<0.001)$ lower than the mean age of men $(44.59 \pm 16.47)$.

Table 2 presents the disease frequencies among study population. Table 3 demonstrates that in compared to lower socioeconomic status, people with higher socioeconomic status significantly less tobacco smoked daily (Table 3). People who had academic studies consumed less tobacco and cigarettes $(\mathrm{p}=0.06$ and $\mathrm{p}=0.03$ ).

The participants consumed vegetables and fruits five or more times in a week, $41.8 \%$ and $27.8 \%$ respectively. People with higher socioeconomic status consumed vegetables, fruits, and fish more than the people with lower socioeconomic status $(p=0.000, p=0.004, p=0.007)$.

People with academic education are less likely to be hypertensive than people with non-academic education (adj. OR: 0.262; CI: 0.161-0.429). Table 3 demonstrates that people with medium socioeconomic status are less likely to be hypertensive than people with high socioeconomic status (adj. OR: 0.426; CI: 0.248-0.734).

Table 4 indicates participants food consumption and their socioeconomic status. Tables 5 and 6 present multivariate logistic regressions assessing the effect of different factors on hypertension and diabetes. Multivariate analyses indicate that people with academic education are less likely to develop diabetes than people with non-academic education (adj. OR: 0.354; CI: 0.177-0.710). People with medium socioeconomic status are less likely to develop diabetes

Table I Demographic Characteristics of the Study Population

\begin{tabular}{|l|c|c|}
\hline Sex & Man & Female \\
\hline Number & $386(32.3 \%)$ & $810(67.7 \%)$ \\
Mean Age & 44.6 & 40.9 \\
\hline
\end{tabular}


Table 2 Frequency of Disease in the Study Population

\begin{tabular}{|l|l|l|l|}
\hline Disease & $\begin{array}{l}\text { Total Number of Cases } \\
\text { (Percentage) }\end{array}$ & $\begin{array}{l}\text { Number of Men with the } \\
\text { Disease }\end{array}$ & $\begin{array}{l}\text { Numbers of Women with the } \\
\text { Disease }\end{array}$ \\
\hline Diabetes & $90(7.53)$ & $20(5.18)$ & $70(8.64)$ \\
Depression & $52(4.35)$ & $10(2.59)$ & $42(5.19)$ \\
Hypertension & $206(17.22)$ & $54(13.99)$ & $152(18.77)$ \\
Traffic injuries & $8(0.67)$ & $5(1.30)$ & $3(0.37)$ \\
Burn & $4(0.33)$ & $I(0.26)$ & $3(0.37)$ \\
Falling & $2(0.17)$ & $I(0.26)$ & $I(0.12)$ \\
Other Disease & $4(0.33)$ & $2(0.52)$ & $2(0.25)$ \\
\hline
\end{tabular}

than people with high socioeconomic status (adj. OR: 0.438; CI: 0.196-0.979).

\section{Discussion}

Today, dramatic changes have occurred in people's life. Malnutrition, unhealthy diet, smoking, alcohol consumption, drug abuse, and stress are unhealthy habits that are considered the dominant forms of lifestyle. ${ }^{7}$ Lifestyle has long been associated with the development of many chronic diseases and NCDs. WHO has identified four major NCDs, ie diabetes, CVDs, cancer, and chronic lung disease/ Chronic Obstructive Pulmonary Disease (COPD) which share common lifestyle-related behavioural risk factors. ${ }^{5}$ In this study, smoking was more prevalent among men than women. $24.30 \%$ of men and $3.27 \%$ of women were daily smokers. The results also showed that daily smoking was the most prevalent in families with lower socioeconomic status and lower education. Similar observations were reported in other studies conducted in Iran and other countries, ${ }^{5,8-10}$ however, they were lower than countries such as Indonesia and Turkey. ${ }^{11,12}$ The study found that the average number of women smokers was higher than men. The per cent of women smokers in Iraq was lower than this study and it was $1.76 \%{ }^{13}$ Although smoking in the present study is high, it was much less than a study using the WHO STEP method in Indonesia, where $54 \%$ of men and $27.6 \%$ smoked daily. ${ }^{12}$ Since the parent's behaviour affect children's health, attitude and performance, some educational and preventative campaigns must be essentially held for parents and their children. Research conducted in Norway found that school anti-smoking programs' efficiency was greatly increased when the parents get involved. ${ }^{14}$ The majority of participants had poor dietary habits. For example, fruits and vegetable intake, the consumption of fish, milk, and dairy were low and inadequate among this study population. These results are comparable with the observations in other studies conducted in Iran and other countries. ${ }^{8,15-18}$ Only $20.35 \%$ of target populations daily consumed fruits and vegetables while, based on the WHO recommendation, at least three servings must be consumed daily as a healthy diet. ${ }^{5}$ According to a study conducted in the United States, diets that included higher dairy intake also help support public health goals. As indicated in the 2010 Dietary Guidelines for Americans (DGA), high dairy products are associated with improved bone health, especially in children and adolescents, and with a reduced risk of CVDs, type 2 diabetes, and lower blood pressure in adults. ${ }^{17}$ The present study showed that only $3.2 \%$ of participants did a level of physical activities in their leisure time. These findings indicated that physical inactivity and sedentary lifestyle was highly prevalent even more among women than men. Similar results were found in

Table 3 Relation Between Socioeconomic Status, Education and Daily Smoking

\begin{tabular}{|c|c|c|c|c|c|c|c|}
\hline \multirow[t]{2}{*}{ Variable } & & \multicolumn{2}{|c|}{ Daily Tobacco Smoking } & \multirow[t]{2}{*}{ p-Value } & \multicolumn{2}{|c|}{ Daily Smoking Cigarette } & \multirow[t]{2}{*}{ p-Value } \\
\hline & & No Smoker & Smoker & & No Smoker & Smoker & \\
\hline \multirow[t]{2}{*}{ Socioeconomic status } & Very low + low & $810(76.42 \%)$ & $98(85.28 \%)$ & \multirow[t]{2}{*}{$0.032 * *$} & $806(77.06 \%)$ & $92(77.97 \%)$ & \multirow[t]{2}{*}{0.8} \\
\hline & Medium + high & $250(23.58 \%)$ & $17(14.78 \%)$ & & $240(22.94 \%)$ & $26(22.03 \%)$ & \\
\hline \multirow[t]{2}{*}{ Education } & Non-Academic & $715(67.77 \%)$ & 89 (77.39\%) & \multirow[t]{2}{*}{$0.03 * *$} & 707 (67.92\%) & $90(76.27 \%)$ & \multirow[t]{2}{*}{0.06} \\
\hline & Academic & 340 (32.23\%) & $26(22.61 \%)$ & & 334 (32.08\%) & 28 (23.73\%) & \\
\hline
\end{tabular}

Note: $*^{*} p<0.05$ 


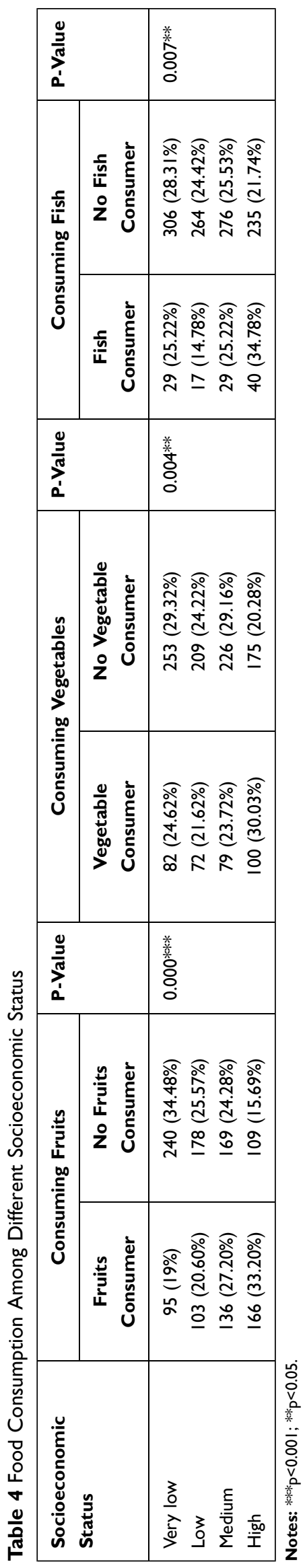

Table 5 Multivariate Logistic Regression Results for Assessing the Effect of Different Factors on Hypertension

\begin{tabular}{|l|c|c|c|}
\hline Factor & Odds Ratio & P-Value & 95\% Conf. Interval \\
\hline Acedu & 0.262 & 0.000 & $0.161-0.429$ \\
Smdaly & 0308 & 0.095 & $0.773-1.228$ \\
Smoke tobacco & 1.633 & 0.490 & $0.405-6.583$ \\
\hline Seat \\
\hline Very low & 1.138 & 0.589 & $0.711-1.825$ \\
Low & 0.597 & 0.050 & $0.370-1.000$ \\
Medium & 0.426 & 0.002 & $0.248-0.734$ \\
\hline
\end{tabular}

Abbreviations: Acedu, academic education; smdaly, smoke daily; sescat, socioeconomic status.

other studies. ${ }^{5,8,19,20}$ The impact of daily lifestyle and low daily activities leads to decreased physical activity directly linked to the prevalence of risk factors such as hypertension and other underlying diseases. In this study, $17.22 \%, 7.53 \%$ and $4.35 \%$ of subjects had hypertension, diabetes and depression. These complications had higher prevalence among women. These findings are parallel to the findings of other studies. ${ }^{21,22}$ A study, conducted on WHO STEP methodology in three Asian and African countries, shows that mean systolic blood pressure (SBP) ranged from 117 to $127 \mathrm{~mm} \mathrm{Hg} .{ }^{23}$ Approximately $60 \%$ and $46 \%$ of the study population had checked their blood pressure and blood sugar, respectively in the past year. In our study, lower socioeconomic groups had a significantly higher prevalence of lifestyle-related risk factors such as smoking and less fruit, vegetables, fish-eating than high socioeconomic groups. ${ }^{15,24-27}$ Variety of diseases (especially lifestylerelated diseases) have in common particular risk factors and are closely associated with socioeconomic status. Women living with lower salaries are usually unemployed,

Table 6 Multivariate Logistic Regression Results for Assessing the Effect of Different Factors on Diabetes

\begin{tabular}{|l|c|c|c|}
\hline Factors & Odds Ratio & P-Value & 95\% Conf. Interval \\
\hline Acedu & 0.354 & 0.003 & $0.177-0.710$ \\
Smdaly & 1.551 & 0.542 & $\begin{array}{l}0.379-6.356 \\
0.746-1.947\end{array}$ \\
Smoke tobacco & 0.381 & 0.246 & $0.626-2.365$ \\
\hline Seat & 1.216 & 0.563 & $0.349-1.489$ \\
\hline Very low & 0.721 & 0.377 & $0.196-0.979$ \\
Low & 0.438 & 0.044 & \\
Medium &
\end{tabular}

Abbreviations: Acedu, academic education; smdaly, smoke daily; sescat, socioeconomic status. 
under-educated, and have much fewer social networks, which may limit their capacity to adopt healthy habits. Underprivileged women considerably have higher health risk factors, such as being overweight or obese, having fewer or no daily consumptions of fruit, and smoking. We need to look at how women can be supported to reduce their contact with chronic disease risk factors. Our findings provide evidence for health policymakers and the level of public health that can examine individuals' healthy lifestyle. This study can be useful considering that appropriate strategies based on people's lifestyle to prevent underlying diseases and control key risk factors are not considered. Also, the existence of native programs for NCDs can be effective in this regard. It seems that in order to link socioeconomic factors with healthy lifestyles, studies should be conducted on a larger scale in order to provide a complete analysis in this field.

\section{Conclusion}

Our study showed that a healthy lifestyle is not practiced satisfactorily and risk factors associated with lifestyle are prevalent among the East-Azerbaijan province population, Iran. Therefore, due to the direct relationship between lifestyle and the development of many chronic diseases and NCDs, it is suggested to organise campaigns and educational seminars to implement healthy lifestyle habits and health promotions programs.

\section{Acknowledgment}

Special thanks to the authors for the questioners and households for their contribution. This research was conducted with the support of Tabriz University of Medical Sciences' health chancellor and was funded by Health Services Management Research Centre, Tabriz University of Medical Science, Tabriz, Iran.

\section{Funding}

No funding was received. However, all authors received their salary from respective universities.

\section{Disclosure}

The authors report no conflicts of interest in this work.

\section{References}

1. Lifestyle. 2019. Available from: http://www.businessdictionary.com/ definition/lifestyle.html. Accessed May 26, 2021.
2. Foroushani AR, Estebsari F, Mostafaei D, et al. The effect of health-promoting intervention on healthy lifestyle and social support in elders: a clinical trial study. Iran Red Crescent Med J. 2014;16(8).

3. Sharma M, Majumdar P. Occupational lifestyle diseases: an emerging issue. Indian J Occup Environ Med. 2009;13(3):109. doi:10.4103/ 0019-5278.58912

4. Ahmadi A, Mobasheri M, Soori H. Prevalence of major coronary heart disease risk factors in Iran. Int J Epidemiol Res. 2014;1:1.

5. Chakma JK, Gupta S. Lifestyle practice and associated risk factors of non-communicable diseases among the students of Delhi University. Int $J$ Health Allied Sci. 2017;6(1):20.

6. Healthy living: what is a healthy lifestyle? Available from: https:// apps.who.int/iris/handle/10665/108180. Accessed May 26, 2021.

7. Farhud DD. Impact of lifestyle on health. Iran J Public Health. 2015;44(11):1442.

8. Sadeghi-Bazargani H, Jafarzadeh H, Fallah M, et al. Risk factor investigation for cardiovascular health through WHO STEPS approach in Ardabil, Iran. Vasc Health Risk Manag. 2011;7:417. doi:10.2147/VHRM.S22727

9. Halimi L, Haghdoost AA, Alizadeh SM. Prevalence of cigarette smoking among Iranian women: a systematic review and meta-analysis. Med J Islam Repub Iran. 2013;27(3):132.

10. Parizadeh D, Momeni AA, Amouzegar A, Azizi F, Hadaegh F. Tobacco smoking: findings from 20 years of the Tehran Lipid and Glucose Study. Int $J$ Endocrinol Metab. 2018;16(4Suppl). doi:10.5812/ijem. 84738

11. Yürekli A, Önder Z, Elibol M. The Economics of Tobacco and Tobacco Taxation in Turkey. Paris, France: International Union Against Tuberculosis and Lung Disease; 2010.

12. Ng N, Stenlund H, Bonita R, Hakimi M, Wall S, Weinehall L. Preventable risk factors for non-communicable diseases in rural Indonesia: prevalence study using WHO STEPS approach. Bull World Health Organ. 2006;84:305-313. doi:10.2471/ BLT.05.023721

13. Al-Badri JHA, Ali MAK, Ali AA, Sahib AJ. Socioeconomic determinants of smoking among Iraqi adults: data from non-communicable risk factor STEPS survey 2015. PLoS One. 2017;12(9):e0184989. doi:10.1371/journal.pone.0184989

14. Murray M, Kiryluk S, Swan A. Relation between parents' and children's smoking behaviour and attitudes. J Epidemiol Community Health. 1985;39(2):169-174. doi:10.1136/jech.39.2.169

15. Esteghamati A, Noshad S, Nazeri A, Khalilzadeh O, Khalili M, Nakhjavani M. Patterns of fruit and vegetable consumption among Iranian adults: a SuRFNCD-2007 study. Br J Nutr. 2012;108 (1):177-181. doi:10.1017/S0007114511005393

16. Ziaei R, Shahi H, Dastgiri S, Mohammadi R, Viitasara E. Fruit and vegetable intake and its correlates among high-school adolescents in Iran: a cross-sectional study. J Public Health (Bangkok). 2019;1-8. doi:10.1093/pubmed/fdz027

17. Esfarjani F, Mohammadi-nasrabadi F, Roustaee R, et al. Household milk consumption and its socio-economic associates in west Azarbayejan province, North-west Iran. Nutr Food Sci Res. 2015;2 (3):21-27.

18. Quann EE, Fulgoni VL, Auestad N. Consuming the daily recommended amounts of dairy products would reduce the prevalence of inadequate micronutrient intakes in the United States: diet modeling study based on NHANES 2007-2010. Nutr J. 2015;14(1):90. doi:10.1186/s12937-015-0057-5

19. Hajian-Tilaki K, Heidari B. Prevalence of obesity, central obesity and the associated factors in urban population aged 20-70 years, in the north of Iran: a population-based study and regression approach. Obes Rev. 2007;8(1):3-10. doi:10.1111/j.1467-789X.2006.00235.x

20. Dunn AL, Andersen RE, Jakicic JM. Lifestyle physical activity interventions: history, short-and long-term effects, and recommendations. Am J Prev Med. 1998;15(4):398-412. doi:10.1016/S0749-3797(98)00084-1 
21. Khalequzzaman M, Chiang C, Choudhury SR, et al. Prevalence of non-communicable disease risk factors among poor shantytown residents in Dhaka, Bangladesh: a community-based cross-sectional survey. BMJ Open. 2017;7(11):e014710. doi:10.1136/bmjopen2016-014710

22. Asgari F, Haghazali M, Heydarian H. Non-communicable diseases risk factors surveillance in Iran. 2009.

23. Tesfaye F, Nawi NG, Van Minh H. Association between body mass index and blood pressure across three populations in Africa and Asia. J Hum Hypertens. 2007;21(1):28-37. doi:10.1038/sj.jhh.1002104

24. Allen L, Williams J, Townsend N, et al. Socioeconomic status and non-communicable disease behavioural risk factors in low-income and lower-middle-income countries: a systematic review. Lancet Glob Health. 2017;5(3):e277-e89. doi:10.1016/S2214-109X(17) 30058-X
25. Ali P, Mojgan G, Mohammad G, Fatemeh N, Marzieh T, Masoumeh S. Association of socioeconomic status and hypertension based on habitual smoking among Iranian population: IHHP study. Acta Biomed. 2018;89(4):498.

26. Connolly V, Unwin N, Sherriff P, Bilous R, Kelly W. Diabetes prevalence and socioeconomic status: a population-based study showing increased prevalence of type 2 diabetes mellitus in deprived areas. $J$ Epidemiol Community Health. 2000;54(3):173-177. doi:10.1136/jech.54.3.173

27. Gamage AU, Jayawardana PL. Knowledge of non-communicable diseases and practices related to healthy lifestyles among adolescents, in state schools of a selected educational division in Sri Lanka. BMC Public Health. 2018;18(1):1-9. doi:10.1186/s12889-017-4622-z
ClinicoEconomics and Outcomes Research

\section{Publish your work in this journal}

ClinicoEconomics and Outcomes Research is an international, peerreviewed open-access journal focusing on Health Technology Assessment, Pharmacoeconomics and Outcomes Research in the areas of diagnosis, medical devices, and clinical, surgical and pharmacological intervention. The economic impact of health policy and health systems

\section{Dovepress}

organization also constitute important areas of coverage. The manuscript management system is completely online and includes a very quick and fair peer-review system, which is all easy to use. Visit http://www.dovepress.com/testimonials.php to read real quotes from published authors. 